\title{
Construction of School Temperature Measurement System with Sensor Network
}

\author{
Ayahiko Niimi, Masaaki Wada, Kei Ito, and Osamu Konishi \\ Department of Media Architecture, Future University-Hakodate \\ 116-2 Kamedanakano-cho, Hakodate 041-8655, Japan \\ \{niimi, wada, kei, okonishi\}@fun.ac.jp
}

\begin{abstract}
We propose the sensor network system using the microcomputer board that can connect to the Internet. This proposed system can acquire information from the sensor of the microcomputer group arranged on the network, and can view collected information on Web browser. In this paper, it is shown to be able to construct easily the microcomputer's sensor network which is combined microcomputer modules (Micro Cube) and the database server and the Web application server. The system that measured the room temperature in school campus was constructed, it has run for four months, and the effectiveness is verified.
\end{abstract}

\section{Introduction}

The digital measurements of the temperature and humidity, etc. become possible, and connecting the system that acquired the measured data on the network becomes possible. However, there are many measurement systems which are rich systems that are used sensors on PC or which are cheap microcomputer systems that need to construct a special network for the sensor network. We propose the sensor network system using the microcomputer board that can connect to the Internet. This proposed system can acquire information from the sensor of the microcomputer group arranged on the network, and can view collected information on Web browser.

In this paper, it is shown to be able to construct easily the microcomputer's sensor network which is combined microcomputer modules (Micro Cube) and the database server and the Web application server. The system that measured the room temperature in school campus was constructed, it has run for four months, and the effectiveness is verified.

The research of ecopic [12] is similar to this research. The ecopic research has aimed the construction of ecopic of the weather observing system that can make it easily by user. Our proposed system has aim of improving the extendibility by using Micro Cube that is generality module.

Chapter 2 describes the sensor module using Micro Cube. Chapter 3 describes the composition of the sensor network. We describe the installation of Micro Cube, the server and the client and the technique of collecting and viewing data. In chapter 4, we describe construction of our proposed system, and discuss about the problem when constructing sensor network. Section 5 describes conclusion and enhancing in a future. 


\section{Sensor Module}

In this section, we describe proposed sensor module.

\subsection{Outline of Micro Cube}

The Micro Cube is a board computer and is composed of several stackable boards 34. Fig. 1 is a photo showing one of the combinations of stacked Micro Cube. The specifications of the CPU and extension boards are summarized in Table 1. It has a CPU board with a RENESAS H8 CPU and a TCP/IP Protocol stack. Stackable boards can vary as follows: Ethernet LAN board, compact flash board, PCMCIA board, serial board (RS232C and RS422) and so on (some boards shown in Fig. 2, 3). Since the different combinations of stackable boards make a seamless connection with the sensors, users can structure an ad hoc sensor network very easily. To get sensor information through the Internet, HTTP is also employed so that user can get data via a standard Web browser.

\subsection{Instrumentation of the Present System}

The Micro Cube used in the system to get the information of room condition is composed of the H8/3069 CPU board, LAN board, and special sensor board.

Table 1. Lineup of the CPU and extension boards of Micro Cube

\begin{tabular}{|l|l|}
\hline Board Name & Features \\
\hline \hline H8/3048BV & CPU board for battery operation \\
H8/3069 & Same for general purpose (see Fig. 2) \\
H8s $/ 2638$ & Same for Controller Area Network (CAN) \\
\hline LAN & Extension board for Ethernet Connection (see Fig. 33) \\
CF & Same for Compact Flash slot \\
IDE & Same for storage devices \\
ADIO & Same for analog/digital IO \\
COM4 & Same for 4-port serial interface \\
RF & Same for wireless communication \\
PCMCIA & Same for PCMCIA slot \\
\hline
\end{tabular}

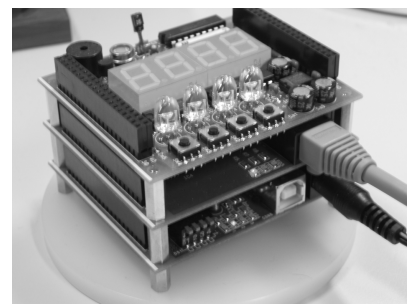

Fig. 1. Photo of a Stacked Micro Cube

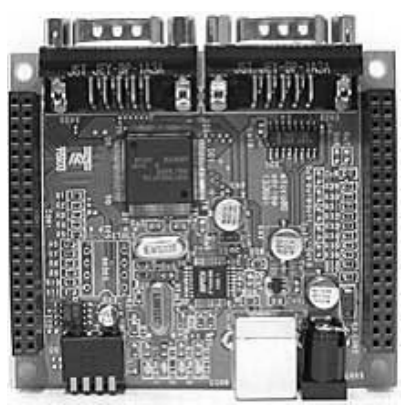

Fig. 2. CPU board 


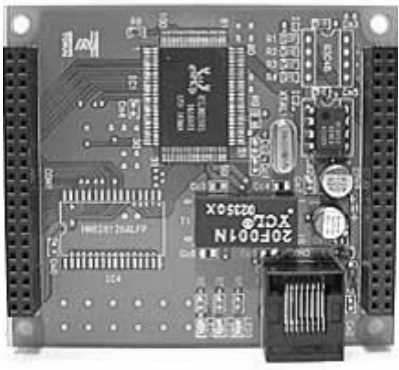

Fig. 3. LAN board

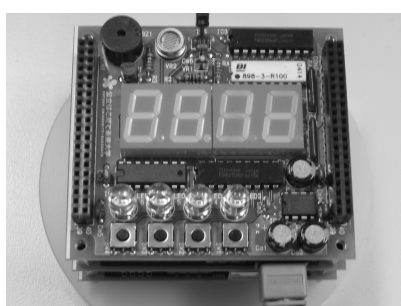

Fig. 4. Sensor board

The special sensor board is utilized the board used of the programming practice class in Future University-Hakodate. (The sensor board is shown in Fig. 4) Future University-Hakodate has the programming practice class with the microcomputer and assembler language as "Media Architecture Practice II". The special board for Micro Cube was designed for its practice class. The push switch, the thermally sensitive resistor (temperature sensor), and CdS sensor (optical sensor) were attached on this board as an input. Moreover, four digits sevensegments LED and four two-color LED were attached as an output. Because an accurate temperature measurement using the thermally sensitive resistor is difficult, a digital sensor is added in this board for our experiment. Humidity can be also measured in this digital sensor. Only the temperature data is acquired this experiment though some sensors are attached on the board. The exchange and the addition of the sensor can be easily done by exchanging the sensor boards.

To confirm the measurement data easily, the measured temperature was displayed in seven-segments LED. Moreover, data can be got by HTTP though the network. When only one sensor module runs, the user can display a present temperature when the user accesses it using Web browser.

\section{Network Configuration}

The sensor network was constructed by using the microcomputer that explained in Chapter 2, Fig. 5 shows the composition of the constructed sensor network system.

The used software is shown below (see table 2). The data store part is implemented by Perl, and the data display part is implemented by JSP.

The flow of the collection of data and the display stored data is shown in Fig. 6. Fig. 6] shows the following steps.

1. Data storage

(a) The Perl script accesses to URL of Micro Cube.

(b) Micro Cube returns the measurement result by HTML format.

(c) HTML is parsed, and necessary data is preserved in the database. 


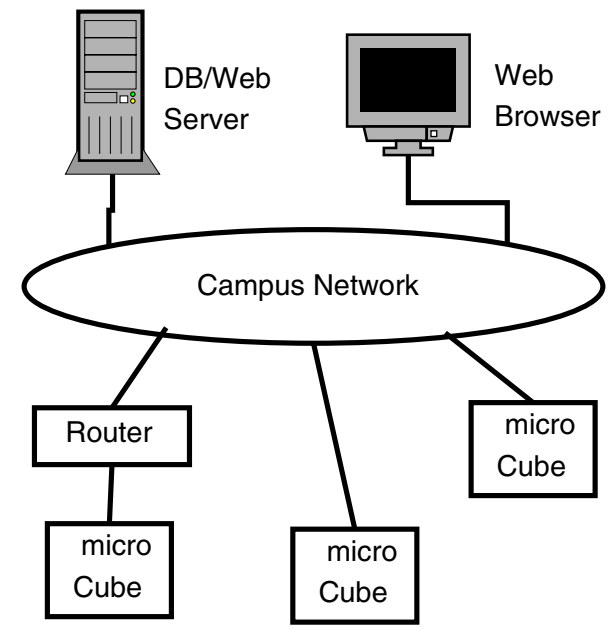

Fig. 5. Network Configuration

Table 2. Software used in the web database server

\begin{tabular}{|l|l|}
\hline System & Software \\
\hline \hline OS & Red Hat Linux release 9 \\
HTTP & Apache 2.0.40 \\
Database & PostgreSQL 7.3.2 \\
Software codes & Tomcat 5.0.28 and Perl 5.8.0 \\
\hline
\end{tabular}

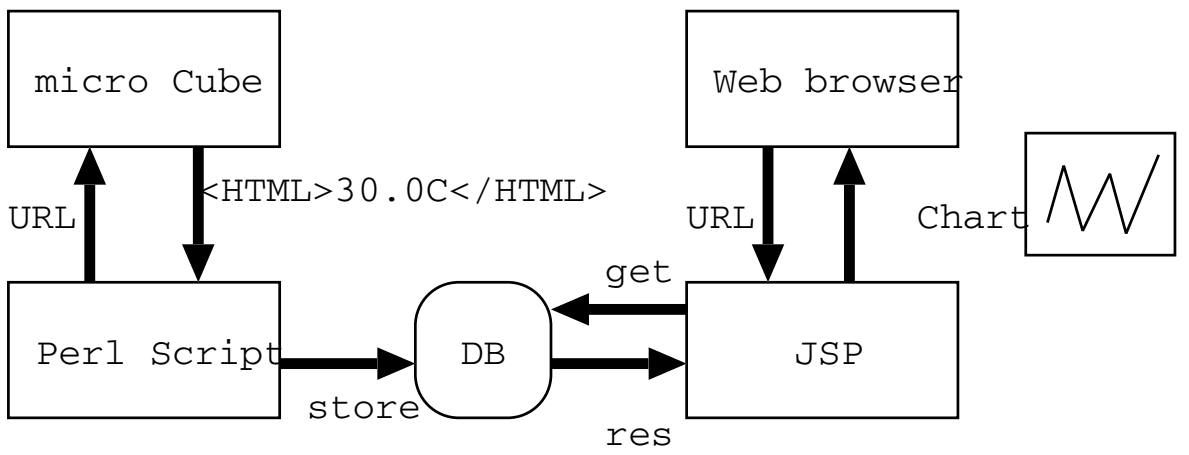

Fig. 6. Flowchart of Proposed System

2. Data browse

(a) URL of the server is opened from a Web browser.

(b) JSP accesses the database.

(c) Necessary data is acquired from the database.

(d) The result is processed to the graph and displayed it on a browser. 
The micro cube arranged in school is connected with campus network (LAN). The data of each sensor module is acquired with the server set up on the campus network at regular intervals, and stores in the database. In this experiment, data is acquired from the sensor module every ten minutes. The acquired data is processed with the Web application server set up on the same server, and can be displayed from Web browser of PC on the campus network.

At first, Micro Cube connected to campus network by arranging it in the router because the router had not been exceeded in LAN of the micro cube. Afterwards, connecting Micro Cube to the campus network even if we modified the program, and the router is not set up became possible so that the router was exceeded.

\section{Experimental Results}

The system that explained in Chapter 3 was actually constructed. The system is constructed in December, 2005, and it is running at March, 2006. Because a lot of modules were able to be reused, the time that had constructed to development was about one week.

Some data display examples are shown as follows. (See Fig. (7 8) The displayed data can be switched to all or a part of room. The displayed range can be switched to a day, a week, a month, or all. Fig. 7 shows all data in one chart, and Fig. 8 shows one data in one chart.

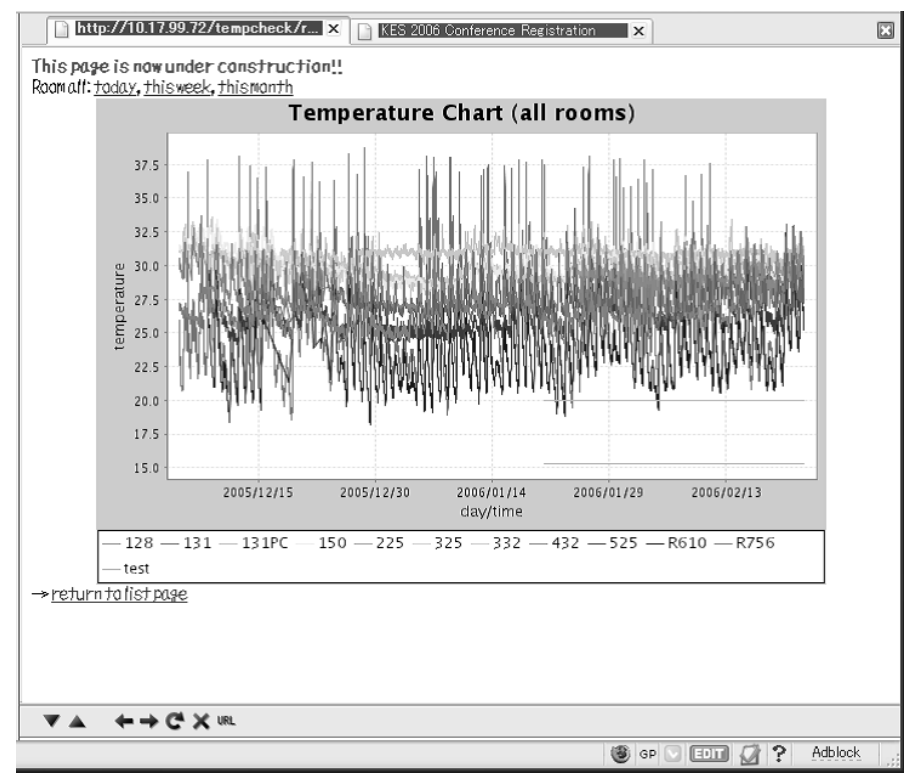

Fig. 7. All Data in One Chart 


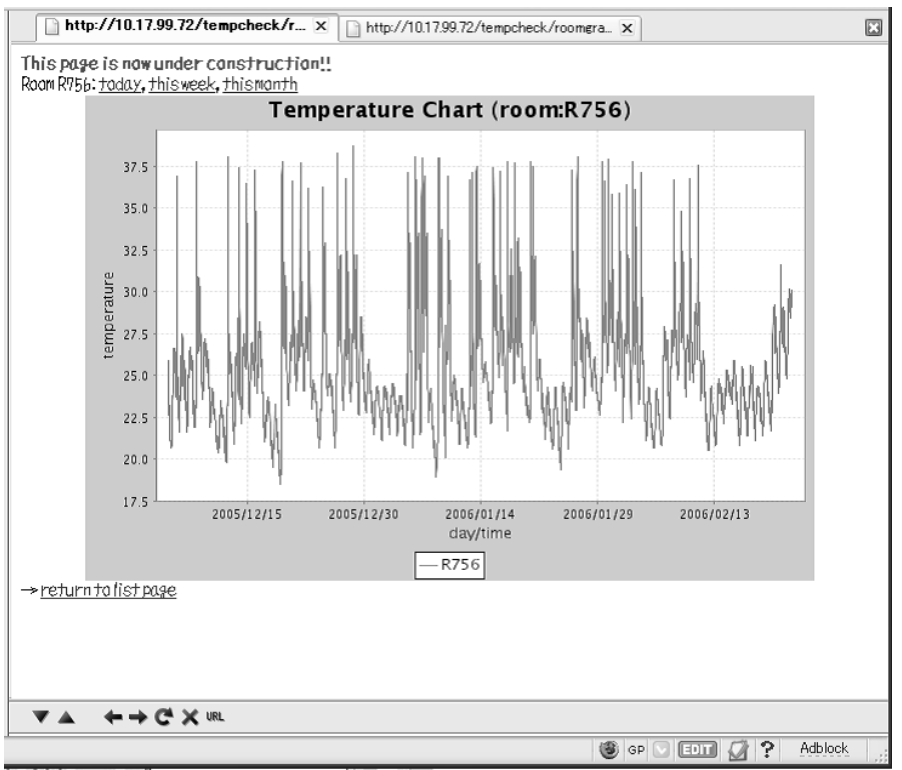

Fig. 8. Selected Data in One Chart

At first, Micro Cube connected to campus network by arranging it in the router because the router had not been exceeded in LAN of the micro cube. However, data might not be able to be acquired normally when some router's passing. Then, connecting Micro Cube to the campus network even if we modified the program, and the router is not set up became possible so that the router was exceeded.

It took time to divide the problem when data was not able to be acquired normally because Micro Cube connected to the campus network. Then, the problem was considered in cooperation with SE that resided in school. For the exceeding router problem, we constructed some environment, such as the dummy server with the PC-UNIX server, passing the router setting, bypassing the router setting, and it undertook the resolution of a problem while capturing the packet that flowed in the network.

Because data began to collect, temperature data is scheduled to be analyzed in the future.

\section{Conclusion}

We proposed the sensor network system using the microcomputer board that can connect to the Internet. This proposed system can acquire information from the sensor of the microcomputer group arranged on the network, and can view collected information on Web browser. In this paper, it was shown to be able to construct easily the microcomputer's sensor network which is combined microcomputer modules (Micro Cube) and the database server and the Web 
application server. The system that measured the room temperature in school campus was constructed, it has run for four months, and the effectiveness was verified. Because data began to collect, temperature data is scheduled to be analyzed in the future. In the analysis of data, it is thought that it is possible to refer to a technique of the multiagent base [56] and an analytical technique of the analysis of the fixed point observation data $7 / 8$.

The system that expands acquired more different type of sensor information will be constructed, and then, obtained data is scheduled to be analyzed in the future. In addition, we want to attach the IR I/O module on the microcomputer board, and to do the research for the ubiquitous computing of the indoor environment controlling.

\section{References}

1. Toda, M., Akita, J., Kimura, K.: Construction of Ubiquitous Weather Observation System. Proc. of The 7th World Multiconference on Systemics, Cybernetics and Informatics (SCI2003), Vol.1 pp.192-197 (2003)

2. Akita, J., Toda, M., Kimura, K.: Handy Meteorological Observation System for Hands-on Study. Proc. of The 8th World Multiconference on Systemics, Cybernetics and Informatics (SCI2004), Vol.X pp.155-159 (2004)

3. Wada. M., Hatanaka, K., Kimura, N., Amagai, K.: Applying an Information Technology in Fishing Industry I. Acquisition of a Three Dimensional Topography. Journal of Japan Institute of Navigation, vol. 112, pp.189-198 (2004) (in Japanese)

4. Hatanaka, K., Wada, M., Kotaki, M.: Instrumentation for the Measurement of Shallow Seabed Topography by a Fishing Echo Sounder. Oceans 2005 MTS/IEEE Conference Proceedings, Ocean Instrumentation I, pp.1-6 (2005)

5. Niimi, A., Konishi, O.: Data Mining for Distributed Databases with Multiagents. KES'2003, Proceedings, Part II, Lecture Notes in Artificial Intelligence 2774, Springer, pp.1412-1418 (2003)

6. Niimi, A., Konishi, O.: Extension of Multiagent Data Mining for Distributed Databases. KES'2004, Proceedings, Part III, Lecture Notes in Artificial Intelligence 3215, Springer, pp.780-787 (2004)

7. Teiten2000 Project, http://www.teiten2000.jp/ (in Japanese)

8. Toda, M., Osanai, A., Niimi, A., Akita, J., Kimura, K., Konishi, O.: Information Access Method to Meteorological Data for Educational Application. World Conference on Education Multimedia, Hypermedia and Telecommunications (ED-MEDIA2005), Association for the Advancement of Computing in Education, Montre'al, Canada, Proceedings, pp.4404-4408 (2005) 